A characterisation of NL using membrane systems without charges and dissolution

Niall Murphy, Damien Woods

Technical Report

NUIM-CS-TR-2008-01
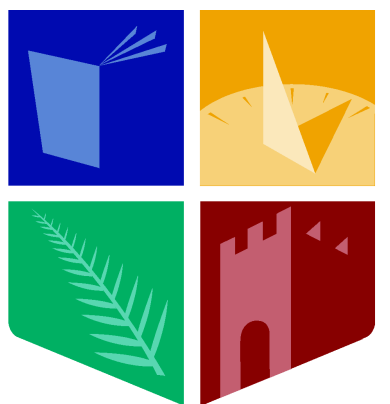

NUI MAYNOOTH

Ollscoil na hÉireann Má Nuad

Department of Computer Science National University of Ireland, Maynooth Ireland 


\title{
A characterisation of NL using membrane systems without charges and dissolution
}

\author{
Niall Murphy ${ }^{1}$ and Damien Woods ${ }^{2}$ \\ 1 Department of Computer Science, National University of Ireland, Maynooth, \\ Ireland \\ nmurphy@cs . nuim.ie \\ 2 Department of Computer Science, University College Cork, Ireland \\ d.woods@cs.ucc.ie
}

\begin{abstract}
We apply techniques from complexity theory to a model of biological cellular membranes known as membrane systems or P-systems. Like circuits, membrane systems are defined as uniform families. To date, polynomial time uniformity was the accepted uniformity notion for membrane systems. Here, we introduce the idea of using $\mathbf{A C}^{0}$ and $\mathbf{L}$ uniformities and investigate the computational power of membrane systems under these tighter conditions. It turns out that the computational power of some systems is lowered from $\mathbf{P}$ to $\mathbf{N L}$, so it seems that our tighter uniformities are more reasonable for these systems. Interestingly, other systems that are known to be lower bounded by $\mathbf{P}$ are shown to retain their computational power under the new uniformity conditions. Similarly, a number of membrane systems that are lower bounded by PSPACE retain their power under the new uniformity conditions.
\end{abstract}

\section{Introduction}

Membrane systems [12] are a model of computation inspired by living cells. In this paper we explore the computational power of cell division (mitosis) and dissolution (apoptosis) by investigating a variant of the model called active membranes [11]. An instance of the model consists of a number of (possibly nested) membranes, or compartments, which themselves contain objects. During a computation, the objects, depending on the compartment they are in, become other objects or pass through membranes. In the active membrane model it is also possible for a membrane to completely dissolve, and for a membrane to divide into two child membranes.

This membrane model can be regarded as a model of parallel computation but it has a number of features that make it somewhat unusual when compared to other parallel models. For example, object interactions are nondeterministic so confluence plays an important role, membranes contain multisets of objects, there are many parameters to the model, etc. In order to clearly see the power of the model we analyse it from the computational complexity point of view, the goal being to characterise the model in terms of the set of problems that it can solve in reasonable time. 
Another, more specific, motivation is the so-called $\mathbf{P}$-conjecture [13] which states that recogniser membranes systems with division rules (active membranes), but without charges, characterise $\mathbf{P}$. On the one hand, it was shown that this conjecture does not hold for systems with non-elementary division as PSPACE upper [16] and lower [1] bounds were found for this variant (non-elementary division is where a membrane containing multiple membranes and objects may be copied in a single timestep). On the other hand, the $\mathbf{P}$-conjecture was shown to hold for all active membrane systems without dissolution rules, when GutiérrezNaranjo et al. [5] gave a $\mathbf{P}$ upper bound. The corresponding $\mathbf{P}$ lower bound (trivially) came from the fact that the model is defined to be $\mathbf{P}$-uniform.

However, here we argue that the aforementioned $\mathbf{P}$ lower bound highlights a problem with using $\mathbf{P}$ uniformity, as it does not tell us whether this membrane model itself has (in some sense) the ability to solve all of $\mathbf{P}$ in polynomial time, or if the uniformity condition is providing the power. In this paper we show that in fact when we use weaker, and more reasonable, uniformity conditions the model does not have the ability to solve all problems in $\mathbf{P}$ (assuming $\mathbf{P} \neq \mathbf{N L}$ ). We find that with either $\mathbf{A} \mathbf{C}^{0}$ or $\mathbf{L}$ uniformity the model characterises $\mathbf{N L}$ in the semi-uniform case, and we give an NL upper bound for the uniform case. We also show that the PSPACE lower and upper bounds mentioned above still hold under these restricted uniformity conditions.

Using the notation of membrane systems (to be defined later) our upper bound on $\mathbf{L}$-uniform and $\mathbf{L}$-semi-uniform membrane systems can be stated as follows.

\section{Theorem 1. $\mathbf{P M C}_{\mathcal{A M}_{-d}^{0}} \subseteq \mathrm{NL}$}

Essentially this theorem states that polynomial time active membrane systems, without dissolution rules, solve no more than those problems in NL. Despite the fact that these systems run for polynomial time (and can even create exponentially many objects), they can not solve all of $\mathbf{P}$ (assuming $\mathbf{N L} \neq \mathbf{P}$ ). This result is illustrated by the bottom four nodes in Figure 1.

The upper bound in Theorem 1 is found by showing that the construction in [5] can be reduced to an instance of the NL-complete problem $s$-t-connectivity (STCON). The full proof appears in Section 3. Next we give a corresponding lower bound.

Theorem 2. $\mathbf{N L} \subseteq \mathbf{P M C}_{\mathcal{A M}_{-d,-u}^{0}}$

To show this lower bound we provide an $\mathbf{A \mathbf { C } ^ { 0 }}$-semi-uniform membrane family that solves STCON. The full proof is in Section 4 and the result is illustrated by the bottom left two nodes in Figure 1. Therefore, in the semi-uniform case we have a characterisation of NL.

\section{Corollary 1. $\mathbf{N L}=\mathbf{P M C}_{\mathcal{A M}_{-d, u}^{0}}$}

We have not yet shown an analogous lower bound result for uniform families. To date our best lower bound is PARITY, which is known not to be in $\mathbf{A C}^{0}$ [4]. We describe this in Section 4.1. 


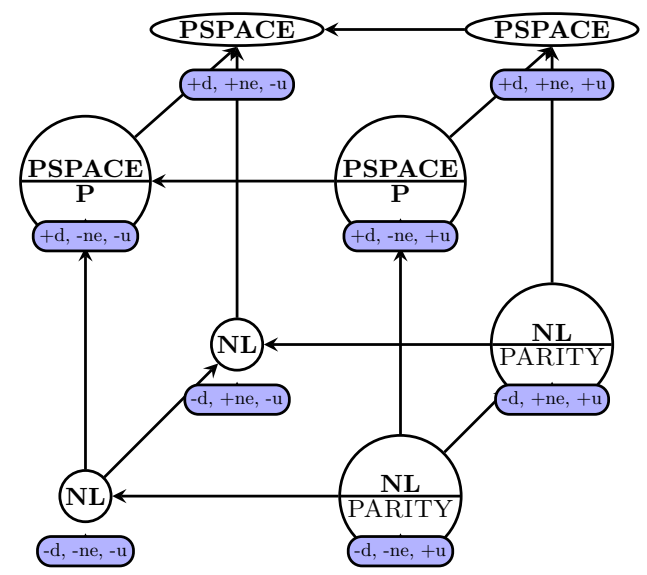

Fig. 1. An inclusion diagram showing the currently known upper and lower bounds on the variations of the model. The top part of a node represents the best known upper bounds, and the lower part the best known lower bounds. An undivided node represents a characterisation.

So far we have shown that four models, that characterise $\mathbf{P}$ when polynomial time uniformity is used, actually only characterise $\mathbf{N L}$ when restricted to be $\mathbf{A} \mathbf{C}^{0}$ uniform. Interestingly, we also show that two other polynomial time uniform membrane system that are known [9] to be lower bounded by $\mathbf{P}$ actually retain this $\mathbf{P}$ lower bound when restricted to be $\mathbf{A} \mathbf{C}^{0}$ uniform. This result is stated as a $\mathbf{P}$ lower bound on membrane systems with dissolution:

Theorem 3. $\mathbf{P} \subseteq \mathbf{P M C}_{\mathcal{A} \mathcal{M}_{+d,+u}^{0}}$

The proof appears in Section 5 and is illustrated by the top front two nodes in Figure 1.

In Section 2.3 we observe that the known PSPACE upper and lower bounds (top four nodes in Figure 1) remain unchanged under $\mathbf{A C}^{0}$ uniformity conditions.

\section{Membrane Systems}

In this section we define membrane systems and complexity classes. These definitions are from Păun [11,12], and Sosík and Rodríguez-Patón [16]. We also introduce the notion of $\mathbf{A} \mathbf{C}^{0}$ uniformity for membrane systems.

\subsection{Recogniser membrane systems}

Active membranes systems are membrane systems with membrane division rules. Division rules can either only act on elementary membranes, or else on both elementary and non-elementary membranes. An elementary membrane is one which does not contain other membranes (a leaf node, in tree terminology). 
Definition 1. An active membrane system without charges is a tuple $\Pi=$ $\left(O, H, \mu, w_{1}, \ldots, w_{m}, R\right)$ where,

1. $m>1$ is the initial number of membranes;

2. $O$ is the alphabet of objects;

3. $H$ is the finite set of labels for the membranes;

4. $\mu$ is a membrane structure, consisting of $m$ membranes, labelled with elements of $H$;

5. $w_{1}, \ldots, w_{m}$ are strings over $O$, describing the multisets of objects placed in the $m$ regions of $\mu$.

6. $R$ is a finite set of developmental rules, of the following forms:

(a) $[a \rightarrow v]_{h}$, for $h \in H, a \in O, v \in O^{*}$

(b) $a[h]_{h} \rightarrow[h b]_{h}$, for $h \in H, a, b \in O$

(c) $\left[{ }_{h} a\right]_{h} \rightarrow[h]_{h} b$, for $h \in H, a, b \in O$

(d) $\left[{ }_{h} a\right]_{h} \rightarrow b$, for $h \in H, a, b \in O$

(e) $\left[{ }_{h} a\right]_{h} \rightarrow\left[{ }_{h} b\right]_{h}\left[{ }_{h} c\right]_{h}$, for $h \in H, a, b, c \in O$.

(f) $\left[h_{o}\left[h_{1}\right]_{h_{1}}\left[h_{2}\right] h_{h_{2}}\left[h_{3}\right]_{h_{3}}\right]_{h_{0}} \rightarrow\left[h_{0}\left[h_{1}\right]_{h_{1}}\left[h_{3}\right] h_{h_{3}}\right]_{h_{0}}\left[h_{0}\left[h_{2}\right]_{h_{2}}\left[h_{3}\right] h_{3}\right] h_{h_{0}}$, for $h_{0}, h_{1}, h_{2}, h_{3} \in H$.

These rules are applied according to the following principles:

- All the rules are applied in maximally parallel manner. That is, in one step, one object of a membrane is used by at most one rule (chosen in a nondeterministic way), but any object which can evolve by one rule of any form, must evolve.

- If at the same time a membrane labelled with $h$ is divided by a rule of type $(e)$ or $(f)$ and there are objects in this membrane which evolve by means of rules of type $(a)$, then we suppose that first the evolution rules of type (a) are used, and then the division is produced. This process takes only one step.

- The rules associated with membranes labelled with $h$ are used for membranes with that label. At one step, a membrane can be the subject of only one rule of types $(b)-(f)$.

In this paper we study the language recognising variant of membrane systems that solves decision problems. A distinguished region contains, at the beginning of the computation, an input - a description of an instance of a problem. The result of the computation (a solution to the instance) is "yes" if a distinguished object yes is expelled during the computation, otherwise the result is "no". Such a membrane system is called deterministic if for each input a unique sequence of configurations exists. A membrane system is called confluent if it always halts and, starting from the same initial configuration, it always gives the same result, either always "yes" or always "no". Therefore, the following interpretation 
holds: given a fixed initial configuration, a confluent membrane system nondeterministically chooses one from a number of valid configuration sequences, but all of them must lead to the same result.

\subsection{Complexity classes}

Here we introduce the notion of $\mathbf{A C}^{0}$ uniformity to membrane systems. Previous work on the computational complexity of membrane systems used (Turing machine) polynomial time uniformity [14]. Consider a decision problem $X$, i.e. a set of instances $X=\left\{x_{1}, x_{2}, \ldots\right\}$ over some finite alphabet such that to each $x_{i}$ there is an unique answer "yes" or "no". We say that a family of membrane systems solves a decision problem if each instance of the problem is solved by some family member. We denote by $\left|x_{i}\right|$ the size of any instance $x_{i} \in X$. AC $\mathbf{C}^{0}$ circuits are DLOGTIME uniform polynomial sized (in input length $n$ ), constant depth, circuits with AND, OR, and NOT gates, and unbounded fan in [3].

Definition 2 ( $\mathrm{AC}^{0}$ uniform families of membrane systems). Let $\mathcal{D}$ be a class of membrane systems and let $f: \mathbb{N} \rightarrow \mathbb{N}$ be a total function. The class of problems solved by uniform families of membrane systems of type $\mathcal{D}$ in time $f$, denoted by $\mathbf{M} \mathbf{C}_{\mathcal{D}}(f)$, contains all problems $X$ such that:

- There exists an $\mathbf{A C}^{0}$ uniform family of membrane systems, $\Pi_{X}=\left(\Pi_{X}(1), \Pi_{X}(2), \ldots\right)$ of type $\mathcal{D}$ : each $\Pi_{X}(n)$ is constructable by an $\mathbf{A C}^{0}$ circuit with unary input $n$.

- Each $\Pi_{X}(n)$ is sound: $\Pi_{X}(n)$ starting with an input (encoded by an $\mathbf{A C}^{0}$ circuit) $x \in X$ of size $n$ expels out a distinguished object yes if an only if the answer to $x$ is "yes".

- Each $\Pi_{X}(n)$ is confluent: all computations of $\Pi_{X}(n)$ with the same input $x$ of size $n$ give the same result; either always "yes" or else always "no".

- $\Pi_{X}$ is $f$-efficient: $\Pi_{X}(n)$ always halts in at most $f(n)$ steps.

Using this definition of $\mathbf{A} \mathbf{C}^{0}$ uniform families, we now define $\mathbf{A C}^{0}$ semiuniform families of membrane systems $\Pi_{X}=\left(\Pi_{X}\left(x_{1}\right) ; \Pi_{X}\left(x_{2}\right) ; \ldots\right)$ as membrane systems whose members $\Pi_{X}\left(x_{i}\right)$ are constructable by $\mathbf{A C}^{0}$ circuits with input $x_{i}$. This is analogous to weakly-uniform circuit families. In this case, for each instance of $X$ we have a special membrane system which therefore does not need a separately constructed input. The resulting class of problems is denoted by $\mathbf{M C}_{\mathcal{D},-u}(f)$. Obviously, $\mathbf{M C}_{\mathcal{D}}(f) \subseteq \mathbf{M C}_{\mathcal{D},-u}(f)$ for a given class $\mathcal{D}$ and a complexity [2] function $f$. Logspace, or $\mathbf{L}$, uniform and semi-uniform families of membrane systems are defined analogously, where we use deterministic logspace Turing machines for the uniformity conditions.

We define $\mathbf{P} \mathbf{M C}_{\mathcal{D}}$ and $\mathbf{P} \mathbf{M C} \mathbf{C}_{\mathcal{D},-u}$ as

$$
\mathbf{P M C}_{\mathcal{D}}=\bigcup_{k \in \mathbb{N}} \mathbf{M C}_{\mathcal{D}}\left(O\left(n^{k}\right)\right), \mathbf{P} \mathbf{M} \mathbf{C}_{\mathcal{D},-u}=\bigcup_{k \in \mathbb{N}} \mathbf{M C}_{\mathcal{D},-u}\left(O\left(n^{k}\right)\right) .
$$

In other words, as the class of problems solvable by uniform (respectively semiuniform) families of membrane systems in polynomial time. We denote by $\mathcal{A M}^{0}$ 
the classes of membrane systems with active membranes and no charges. We denote by $\mathcal{A M}_{-n e}^{0}$ the classes of membrane systems with active membranes and only elementary membrane division and no charges. We denote by $\mathcal{A M}_{+n e}^{0}$ the classes of membrane systems with active membranes, and both non-elementary and elementary membrane division and no charges. We denote by $\mathbf{P} \mathbf{M C}_{\mathcal{A M}_{-d}^{0}}$ the classes of problems solvable by uniform families of membrane systems in polynomial time with no charges and no dissolution rules.

In this paper we are using DLOGTIME- $\mathbf{A C}^{0}$ uniformity which can somewhat cumbersome to analyse, therefore in our proofs we use an $\mathbf{A C}^{0}$ equivalent model called the CRAM [6].

Definition 3 (CRAM). A CRAM [6] is a constant time PRAM with polynomial processors. Each processor is able to shift a word in memory by a polynomial number of bits.

\section{3 $\quad \mathrm{AC}^{0}$ uniformity and PSPACE results}

Membrane systems with active membranes, without charges, and using nonelementary division have been shown to characterise PSPACE $[1,16]$. For the lower bound, a $\mathbf{P}$-uniform membrane system is given [1] that solves instances of QSAT in polynomial time. Clearly, stricter uniformity notions have no affect on the PSPACE upper bound. We now show that the use of $\mathbf{A \mathbf { C } ^ { 0 }}$ uniformity does not change this lower bound.

The uniformity machine inputs the numbers $n$ and $m$ representing the number of variables and clauses of the QSAT instance, and uses them to construct a polynomial number of objects, rules and membranes. We observe that the construction in [1] is in $\mathbf{A C}^{0}$, the most complicated aspect involves multiplication by constants (essentially addition) which is known [7] to be in $\mathbf{A C}^{0}$. Although we omit the details, it is not difficult to see that a CRAM constructs the membrane system in constant time from $n$ and $m$. Similarly, the encoding of the instance as objects to be placed in the input membrane involves only addition.

\section{NL Upper bound on active membranes without dissolution rules}

Previously the upper bound on all active membrane systems without dissolution was $\mathbf{P}$ [5]. As an aside, we remark that this is a very enlightening proof since it first highlighted the importance of dissolution. Without dissolution, membrane division, even non-elementary division, can be modelled as a special case of object evolution. It is also worth noting that these systems can create exponential numbers of objects, yet they can not compute anything outside $\mathbf{P}$.

Since membrane systems are usually $\mathbf{P}$-uniform, this $\mathbf{P}$ upper bound was considered a characterisation of $\mathbf{P}$. However, having a lower bound of the same power as the uniformity condition is somewhat unsatisfactory, as it tells us little about the computing power of the actual membrane system itself. In this section 
we will show that if we tighten the uniformity condition to be $\mathbf{A C}^{0}$, or even $\mathbf{L}$, it is possible to decide in NL whether or not the system accepts. We give an overview rather than the full details.

The proof of the $\mathbf{P}$ upper bound in [5] involves the construction of a dependency graph representing all possible computation paths of a membrane system on an input. The dependency graph for a membrane system $\Pi$ is a directed graph $G_{\Pi}=\left(V_{\Pi}, E_{\Pi}\right)$. Each vertex $a$ in the graph is a pair $a=(v, h) \in \Gamma \times H$, where $\Gamma$ is the set of objects and $H$ is the set of membrane labels. An edge connects vertex $a$ to vertex $b$ if there is an evolution rule such that the left hand side of the rule has the same object-membrane pair as $a$ and the right has an object-membrane pair matching $b$.

If we can trace a path from the vertex (yes, env) (indicating an accepting computation) back to a node representing the input it is clear that this system must be an accepting one. It is worth noting that, unlike upper bound proofs for a number of other computational models, the dependency graph does not model entire configuration sequences, but rather models only those membranes and objects that lead to a yes output.

The original statement of the proof constructed the graph in polynomial time and a path was found from the accepting node to the start node in polynomial time. We make the observation that the graph $G_{\Pi}$ can be constructed in deterministic logspace, and even in $\mathbf{A} \mathbf{C}^{0}$. We omit the details, but our claim can be verified by checking that the construction in [5] is indeed $\mathbf{A \mathbf { C } ^ { 0 }}$ uniform. Also we note that the problem of finding a path from the accepting vertex to one of the input vertices is actually an instance of MSTCON, a variation of the NL-complete problem STCON. STCON is also known as PATH [15] and REACHABILITY [10].

Definition 4 (STCON). Given a directed graph $G=(V, E)$ and vertices $s, t \in$ $V$, is there a directed path in $G$ from $s$ to $t$ ?

Definition 5 (MSTCON). Given a directed graph $G=(V, E)$, vertex $t \in V$ and $S=\left\{s_{1}, s_{2}, \ldots, s_{|G-1|}\right\} \subseteq V$, is there a path in $G$ from $t$ to any element of $S$ ?

MSTCON is NL-complete as a logspace machine can add a new start vertex $s^{\prime}$, with edges from $s^{\prime}$ to each vertex in $S$, to give an instance of STCON.

Since we have shown that the problem of simulating a membrane system without charges and without dissolution can be encoded as an NL-complete problem we have proved Theorem 1. The proof holds for both $\mathbf{A} \mathbf{C}^{0}$ and $\mathbf{L}$ uniformity, as well as both uniform and semi-uniform membrane systems without dissolution.

\section{NL lower bound for semi-uniform active membranes without dissolution}

Here we provide a proof of Theorem 2 by giving a membrane system that solves STCON in a semi-uniform manner. 
The algorithm works by having each edge in the problem instance graph represented as a membrane. An $s$ object is placed in the $s$ membrane. Multiple copies of the object move from membrane to membrane following (or simulating) each different path through the graph in parallel. If an object enters the $t$ membrane, the system outputs a yes object and halts. Otherwise, a no object is output from the system.

We now give a proof of Theorem 2 .

Proof. Each instance of the problem STCON is a tuple $((V, E) s, t)$. We let $n$ and $m$ be the number of vertices and edges in the graph respectively. We assume an ordering on instances (say by $n$ and then lexicographically). We define a function $f(k)$, computable in $\mathbf{A} \mathbf{C}^{0}$, that maps the $k^{\text {th }}$ instance to the following membrane system $\Pi_{k}$.

- The set of labels $H=\{$ input, output, count, s, t $\}$,

- The initial membrane structure is, where $e_{i} \in E, \mu=$ [env $\left[\right.$ input $\left.[\mathrm{s}][\mathrm{t}]\left[e_{1}\right] \ldots\left[e_{|E|}\right]\right]$ [output $][$ count $\left.]\right]$.

- The working objects $\Gamma=\{$ yes, no $\} \cup\left\{c_{i}: i \in\{1,2, \ldots, 2 n+1\}\right\} \cup\left\{v_{i}: \forall v_{i} \in V\right\}$.

- The initial multisets are all empty except $\mathcal{M}_{\text {count }}=\left\{c_{2 n+1}\right\}$.

In the input membrane we place the object node given by $s$.

The evolution rules are as follows. For each vertex we will create a rule so that if an object representing that vertex is outside a membrane that represents an edge leaving that vertex, it will be communicated inside that edge membrane.

$$
v_{i}\left[\left(v_{i}, v_{j}\right)\right] \rightarrow\left[\left(v_{i}, v_{j}\right) v_{i}\right]: \forall v_{i}, v_{j} \in V
$$

Once an object has started on an edge we want it to pass out again marked with the destination vertex. We make $|E|$ copies of the object to ensure that there will be at least one object for each node in the next timestep. We also exclude rules for the $t$ membrane, this is to ensure that an object will not leave without signalling the end of the computation.

$$
\left[{ }_{\left(v_{i}, v_{j}\right)} v_{i}\right] \rightarrow\left[\left(v_{i}, v_{j}\right)\right] v_{j}^{|E|}: \forall v_{i}, v_{j} \in V \backslash\{t\}
$$

When the object $t$ is in the edge membrane leading to $t$ we want it to exit as a yes object.

$$
\left[\left(v_{i}, t\right) v_{i}\right] \rightarrow\left[\left(v_{i}, t\right)\right] \text { yes }: \forall v_{i} \in V \backslash\{t\}
$$

Then we want to send it out to the environment.

$$
\text { [input yes }] \rightarrow[\text { input }] \text { yes }
$$

We also have a counter that counts down in parallel with the above steps.

$$
\left[\text { count } c_{i} \rightarrow c_{i-1}\right]: i \in\{1,2, \ldots, 2 n+1\}
$$

If we output a yes, this occurs on or before timestep $2 n$. Therefore, when the counter reaches zero, there must not have been a yes object, so we output a no.

$$
\left[\text { count } c_{0}\right] \rightarrow[\text { count }] \text { no }
$$


And send it to the environment.

$$
[\text { input no }] \rightarrow[\text { input }] \text { no }
$$

The membrane system can be constructed by a CRAM in constant time and so is $\mathbf{A \mathbf { C } ^ { 0 }}$ uniform. Note that we encode the edges of the graph as membranes, rather than objects. In the membrane computing framework, for uniform membrane systems, inputs must be specified (encoded) as objects. Therefore our algorithm is semi-uniform as we require a different membrane system for each unique problem instance.

If we relax the definition of uniformity so that the inputs may be specified as membranes inside the input membrane, then our membrane system would be uniform. This would give a more general result, however it is not permitted within the membrane framework that we are analysing.

\subsection{PARITY lower bound for uniform active membranes without dissolution}

The previous proof gave a lower bound for a semi-uniform membrane system. Here we consider the uniform case. We show that PARITY $\in \mathbf{P M C}_{\mathcal{A M}_{-d,+u}^{0}}$ by providing an $\mathbf{A C}^{0}$ uniform membrane system that can solve instances of the problem. PARITYis the problem of telling whether the number of 1 symbols in the input word is odd. This problem is known [4] to be outside of $\mathbf{A} \mathbf{C}^{0}$, and so $\mathbf{A C}^{0}$ is a reasonable uniformity measure in this case.

Our uniformity machine takes as input $n \in \mathbb{N}$ and constructs a set of objects $\left\{\operatorname{odd}_{1^{i} 0^{j}}\right\} \cup\left\{\right.$ even $\left._{1^{i} 0^{j}}\right\} \forall i, j$ such that $i+j=n$. Objects yes and no are also created. A type $(a)$ rule is created mapping every odd object with $i$ " 1 " symbols to the even object with $i-1$ " 1 " symbols in it. A type (a) rule is created mapping every even object with $i$ " 1 " symbols to the odd object with $i-1$ "1" symbols in it. A rule is created from object odd $00 \ldots 0$ to yes and from even $00 \ldots 0$ to no.

The $\mathbf{A C}^{0}$ uniformity machine (a CRAM) rearranges the input word $w$ by moving all 1 symbols to the left and all 0 symbols to the right, to give $w^{\prime}$. Then the symbol even $w^{\prime}$ in placed in the input membrane.

As the system runs, the initial object evolves alternately between odd and even until only 0 symbols are left in the subscript, then a yes (or no) will be evolved indicating the input word contained an odd (or even) number of 1 symbols.

\section{$5 \quad \mathrm{P}$ lower bound on uniform families of active membrane systems with dissolving rules}

So far we have seen that by tightening the uniformity condition from $\mathbf{P}$ to $\mathbf{A C}^{0}$ we lower the power of some models from $\mathbf{P}$ down to $\mathbf{N L}$ (See Figure 1). In this section we show that does not happen for all models with at least $\mathbf{P}$ power. 
More precisely, we prove Theorem 3 by showing that $\mathbf{A C}^{0}$ uniform, polynomial time, membrane systems with dissolution are lower bounded by $\mathbf{P}$. Naturally this result also holds for the semi-uniform case.

Proof. A constant time CRAM encodes an instance of the Circuit VALUE problem (CVP) [8] as a $\mathbf{P} \mathbf{M C}_{\mathcal{A} \mathcal{M}_{+d,+u}^{0}}$ membrane system using the gadget membranes and rules shown in Figure 2. The figure shows AND and OR gadgets, a NOT gadget can be made with the rules $\left[{ }_{\text {not }} T\right] \rightarrow\left[{ }_{\text {not }}\right] F,\left[{ }_{\text {not }} F\right] \rightarrow\left[{ }_{\text {not }}\right] T$. The resulting membrane system directly solves the instance of CVP in polynomial time.

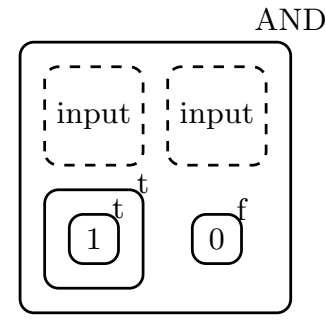

$T\left[{ }_{t}\right] \rightarrow\left[{ }_{t} T\right]$

$\left[{ }_{t} T\right] \rightarrow \lambda$

$F[f] \rightarrow\left[{ }_{f} F\right]$

$\left[\begin{array}{ll}{ }_{f} & F] \rightarrow \lambda\end{array}\right.$

$[$ AND 1$] \rightarrow[$ AND $] T$

$[$ AND 0$] \rightarrow[$ AND $] F$

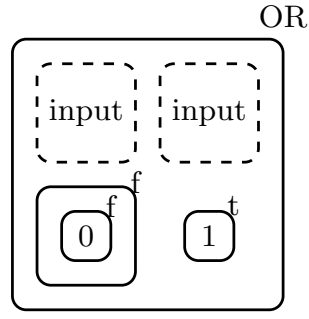

$F[f] \rightarrow[f F]$

$[f F] \rightarrow \lambda$

$T[t] \rightarrow\left[{ }_{t} T\right]$

$\left[{ }_{t} T\right] \rightarrow \lambda$

$[\mathrm{Or} \quad 0] \rightarrow[\mathrm{OR}] F$

$[\mathrm{OR} 1] \rightarrow[\mathrm{OR}] T$

Fig. 2. AND and OR gadgets which can be nested together to simulate a circuit. Here "input" is either $T, F$, or a nested gadget membrane.

To ensure uniformity we have an input membrane (inside the skin membrane) where the initial input assignments for each variable are placed. For example if input gate $i$ is true and input gate $j$ is false we would have input objects $T_{i}$ and $F_{j}$ in the input membrane. When the computation starts the truth assignments descend into the encoded circuit until they reach their appropriate "input gate" gadget where they start the computation. We simulate multiple fanouts by outputting multiple copies of the resulting truth value of each gate. We also give each gadget a unique label and the output of each gate would be tagged. The output of a gate moves up through the layers of the membrane system until it reaches the correct gate according to its tag.

\section{Acknowledgements}

Niall Murphy is funded by the Irish Research Council for Science, Engineering and Technology. Damien Woods is funded by Science Foundation Ireland grant number 04/IN3/1524. 


\section{References}

1. A. Alhazov and M. de Jesús Pérez-Jiménez. Uniform solution to QSAT using polarizationless active membranes. In J. Durand-Lose and M. Margenstern, editors, Machines, Computations and Universality (MCU), volume 4664 of LNCS, pages 122-133, Orléans, France, Sept. 2007. Springer.

2. J. L. Balcázar, J. Diaz, and J. Gabarró. Structural complexity I. Springer-Verlag New York, Inc., New York, NY, USA, 2nd edition, 1988.

3. D. A. M. Barrington, N. Immerman, and H. Straubing. On uniformity within $\mathrm{NC}^{1}$. Journal of Computer and System Sciences, 41(3):274-306, 1990.

4. M. L. Furst, J. B. Saxe, and M. Sipser. Parity, circuits and the polynomial-time hierarchy. Theory of Computing Systems (formerly Mathematical Systems Theory), 17(1):13-27, 1984.

5. M. A. Gutiérrez-Naranjo, M. J. Pérez-Jiménez, A. Riscos-Núñez, and F. J. RomeroCampero. Computational efficiency of dissolution rules in membrane systems. International Journal of Computer Mathematics, 83(7):593-611, 2006.

6. N. Immerman. Expressibility and parallel complexity. SIAM Journal on Computing, 18(3):625-638, 1989.

7. R. M. Karp and V. Ramachandran. Parallel algorithms for shared memory machines. In J. van Leeuwen, editor, Handbook of Theoretical Computer Science, volume A, chapter 17, pages 869-941. Elsevier, Amsterdam, 1990.

8. R. E. Ladner. The circuit value problem is log space complete for P. SIGACT News, 7(1):18-20, 1975.

9. N. Murphy and D. Woods. Active membrane systems without charges and using only symmetric elementary division characterise P. In G. Eleftherakis, P. Kefalas, and G. Păun, editors, Proceedings of the 8th Workshop on Membrane Computing, Thessaloniki, Greece, pages 455-470, June 2007.

10. C. H. Papadimitriou. Computational Complexity. Addison Wesley, November 1993.

11. G. Păun. P Systems with active membranes: Attacking NP-Complete problems. Journal of Automata, Languages and Combinatorics, 6(1):75-90, 2001. and CDMTCS TR 102, Univ. of Auckland, 1999 (www.cs. auckland.ac.nz/CDMTCS).

12. G. Păun. Membrane Computing. An Introduction. Springer-Verlag, Berlin, 2002.

13. G. Păun. Further twenty six open problems in membrane computing. In Proceedings of the Third Brainstorming Week on Membrane Computing,Sevilla(Spain), January 31st - February 4th, pages 249-262, 2005.

14. M. J. Pérez-Jiménez, A. Romero-Jiménez, and F. Sancho-Caparrini. Complexity classes in models of cellular computing with membranes. Natural Computing, 2(3):265-285, August 2003.

15. M. Sipser. Introduction to the Theory of Computation. PWS Publishing Company, 1996.

16. P. Sosík and A. Rodríguez-Patón. Membrane computing and complexity theory: A characterization of PSPACE. Journal of Computer and System Sciences, 73(1):137-152, 2007. 